\title{
Triterpenos e atividade carrapaticida da espécie Lecythis usitata (Lecythidaceae).
}

\author{
Triterpenes and acaricide activity of the species Lecythis usitata \\ (Lecythidaceae).
}

Sinval Garcia Pereira ${ }^{1}$, Giselle Maria Skelding Pinheiro Guilhon ${ }^{2}$

${ }^{1}$ Universidade de Itaúna, Itaúna, MG - Brasil. ${ }^{1}$ Universidade Federal do Maranhão, Centro de Ciências Agrárias e Ambientais (UFMA-CCAA), Chapadinha, MA - Brasil.

${ }^{2}$ Universidade Federal do Pará, Instituto de Ciências Exatas e Naturais (UFPA/ICEN), Belém do Pará, PA Brasil.

\section{Resumo}

Introdução: A resistência do Rhipicephalus microplus (carrapato do gado) aos acaricidas sintéticos deu origem à necessidade de novas investigações científicas sobre formas alternativas de controlá-lo. Objetivos: Dessa forma, o objetivo deste trabalho foi realizar a investigação química de folhas de Lecythis usitata e avaliar o efeito acaricida sobre larvas e fêmeas de $R$. microplus dos extratos, frações e substâncias identificadas. Metodologia: Os extratos foram obtidos das folhas de L. Usitata, inicialmente extraiu com hexano e, posteriormente com metanol. O extrato de metanólico (PFQP) foi particionado, obtendo-se fração acetato de etila (PFQO) que foi fracionada por procedimentos cromatográficos produzindo triterpenos. O efeito acaricida foi avaliado contra $R$. microplus usando o teste de pacotes larvais e teste de imersão de fêmeas. Resultados: O melhor resultado foi observado para o PFQO que causou $97 \%$ de mortalidade quando uma solução de $20 \%$ foi usada. Na mesma concentração, o extrato polar PFQP mostrou 91\% de mortalidade. Conclusão: Os efeitos biológicos variaram de acordo com os extratos e as concentrações das frações. O Friedelanol, como principal composto identificado, e mistura dos triterpenos $\alpha$-amirina e $\beta$-amirina, taraxerol e lupeol foram inativos, considerando os parâmetros e concentrações testados.

Palavras - chaves: Friedelanol; Rhipicephalus microplus; Sapucaia.

Autor correspondente:

Sinval Garcia Pereira

Endereço: Rua projeta 2, $n^{\circ}$ 25, Boa Vista,

Recebido em: 24/06/2020

CEP: 655000-000 - Chapadinha (MA), Brasil.

Revisado em: 05/08/2020

Aceito em: $17 / 08 / 2020$

E-mail: sinval.garcia@ufma.br

Publicado em: 31/08/2020 


\section{Abstract}

Introduction: Resistance of Rhipicephalus microplus (Cattle tick) to synthetic acaricide has given rise to the need of new scientific investigations on alternative ways to control it. Objectives: The objective of this work was to carry out the chemical investigation of Lecythis usitata leaves and to evaluate the acaricide effect on R. microplus larvae and females of extracts, fractions and identified substances from this species. Methodology: The leaves of $L$. usitata we extracted under with hexane and subsequently, with methanol. The methanol extract (PFQP) was partitioned giving an ethyl acetate fraction (PFQO) that was fractionated by chromatographic procedures yielding triterpenes. The acaricidal effect was evaluated against $R$. microplus using the adult immersion tests and larval packet tests. Results: The best result was observed for the PFQO that cause $97 \%$ of mortality when a $20 \%$ solution was used. At the same concentration the polar extract PFQP showed $91 \%$ of mortality. Conclusion: The biological effects varied according to the extracts and fraction concentrations. Friedelanol, as the major identified compound, and mixture, of the triterpenes $\alpha$-amyrins and $\beta$-amyrins, taraxerol and lupeol were inactive, considering the tested parameters and concentrations.

Keywords: Friedelanol; Rhipicephalus microplus; Sapucaia nut.

\section{Introdução}

Os triterpenos são um dos grupos de metabólitos secundários de plantas mais divesificados, são componentes de ceras de superfície, de membranas especializadas e podem atuar como moléculas sinalizadoras, enquanto os triterpenes glicosilados fornecem proteção contra patógenos e pragas e têm uma ampla aplicação nos setores de alimentos, saúde e biotecnologia industrial ${ }^{1}$.

Nas investigações químicas das espécies da família Lecythidaceae, à qual pertence à espécie Lecythis usitata, destacam-se exatamente os metabólitos secundários pertencentes à classe dos terpenos ${ }^{2,3,4} \mathrm{e}$ nos estudos biológicos, ações antiartrítica, antifúngica, antiprurítica $^{5,6,7}$, cicatrizante de feridas tópicas ${ }^{8}$, dor e inflamação ${ }^{9}$ são citadas.

A espécie L. usitata conhecida como castanhasapucaia ou sapucaia caracteriza-se como árvore mediana até grande porte; com flores violáceo-pálidas, esbranquiçadas, quando velhas, odoríferas e sementes comestíveis (FIGURA 1) como em Lecythis pisonis ${ }^{10}$.

A espécie divide-se em duas variedades cujos habitats são bem diversos; a de maior porte é a variedade paraensis, explorada comercialmente ${ }^{11}$. Lecythidaceae tem concentração maior na região neotropical ${ }^{12,13}$ e forma possivelmente um táxon monofilético ${ }^{14}$. No Brasil, o gênero Lecythis é o mais comum e caracteriza-se pela peculiaridade de seus frutos na forma de urnas, de casca dura e de aparência lenhosa, e que produzem uma boa quantidade de amêndoas comestíveis ou não ${ }^{15}$.

Estudos demonstram que as plantas com seus metabólitos secundários podem ser uma alternativa promissora no controle do Rhipicephalus microplus e, já com destaque as espécies timbó (Dahlstedtia pentaphylla) $)^{16,17}$, nim (Azadirachta indica A. Juss. $)^{18,19}$, eucalipto (Eucaliptus spp. $)^{20,21}$, sabonete de soldado (Sapindus saponaria) $)^{22}$, citronelas (Cymbopogon winterianus e Cymbopogon citratus) $)^{23,24}$ e também as espigas de pimenta-de-macaco (Piper tuberculatum) como larvicida ${ }^{25}$.

Os mais recentes grupos químicos de produtos contra os carrapatos que se encontram disponíveis no mercado são: as formamidinas, as avermectinas, os organofosforados ${ }^{26}$ e os piretróides, compostos sintéticos que apresentam estruturas semelhantes à piretrina, substância existente nas flores do Chrysanthemum (Pyrethrun) cinenarialfolium ${ }^{27}$. Algumas dessas bases 
referidas têm restrição ao uso em animais durante o período de lactação ${ }^{28,29,30}$, o que é agravado pelo manejo inadequado dos carrapaticidas, acelerando a seleção de carrapatos resistentes aos princípios ativos disponíveis comercialmente aumentando os riscos e os custos

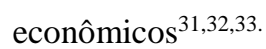

A perda econômica anual devido aos parasitos internos e externos dos bovinos no Brasil é estimada em dólares e é de pelo menos $\$ 13,96$ bilhões. O carrapato bovino ( $R$. microplus) causa uma perda de $\$ 3,24$ bilhões anuais, considerando-se o número total de animais em risco e os efeitos negativos do parasitismo sobre a produtividade do gado. Essa informação deve ser considerada pelos tomadores de decisão para influenciar programas de investigação e regulação a fim de desenvolver políticas sustentáveis que reduzam o impacto do parasitismo sobre a rentabilidade dos pecuaristas brasileiros ${ }^{34}$.

\section{Metodologia}

\section{Coleta e identificação da planta}

Folhas de L. usitata (FIGURA 1) foram coletadas no Cerrado leste do Maranhão, localizado no Município de Chapadinha - MA e, posteriormente, foram secas à temperatura ambiente, em local coberto e arejado e trituradas em moinho tipo faca. A identificação foi por comparação a uma exsicata da planta depositada no Herbário do Museu Paraense Emílio Goeldi em Belém PA (MPEG): L. usitata Miers. $\quad \mathrm{N}^{\circ} 200418$ (Lecythidaceae).
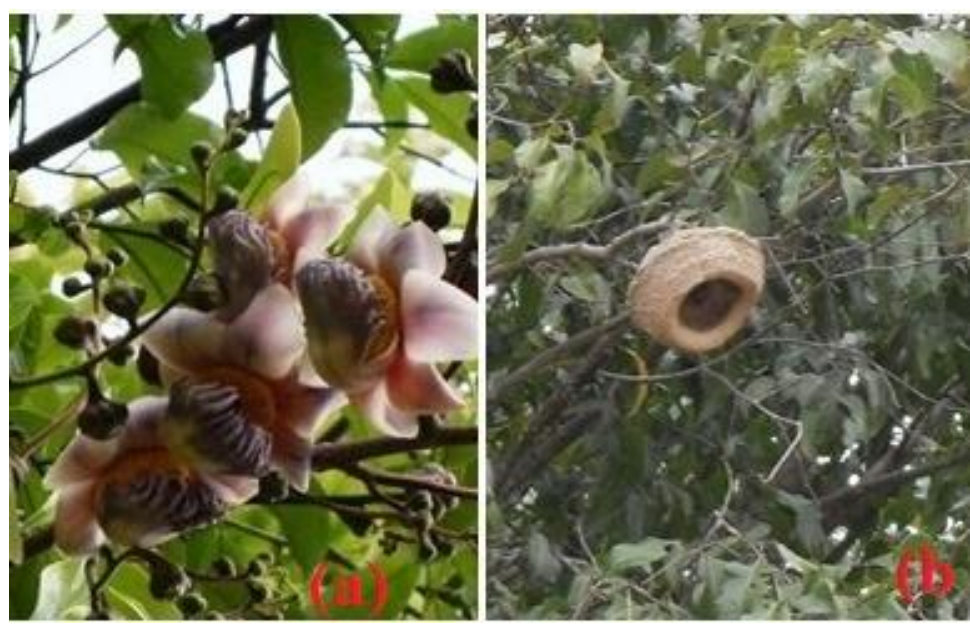

FIGURA 1 - Lecythis usitata em época de inflorescência, ramo florido(a) e fruto(b) aberto mostrando por onde são liberadas as amêndoas (Fotografia tirada no Município de Chapadinha-MA)

\section{Obtenção dos extratos, frações e substâncias}

Para o estudo químico e biológico foram utilizados $2.455 \mathrm{~g}$ de folhas secas e trituradas de L. usitata. Esse material foi submetido à extração sob refluxo, a primeira extração foi realizada com hexano durante duas horas com temperatura máxima de $55^{\circ} \mathrm{C}$. Após a filtração a solução hexânica foi concentrada em evaporador rotativo resultando no extrato hexânico (PFQA - 132 g) e o resíduo, que foi extraído com metanol nas mesmas condições, obtendo-se o extrato metanólico (PFQP - 86 g).
O extrato metanólico foi submetido à partição utilizandose uma parte de água destilada para três de acetato de etila PA, o que resultou, após a concentração em evaporador rotativo, a fração orgânica (PFQO - 57 g), utilizada no estudo químico, a fração aquosa foi descartada. Parte da fração orgânica (PFQO - 31g) foi submetida à cromatografia em coluna (CC) e obtiveram-se 31 subfrações, as quais foram monitoradas por CCD e reunidas ( 1 a 6 , de 7 a 10, de 11 a 17 e de 18 a 23, de 24 a 31) e purificadas por recristalização em misturas de hexano, acetato de etila e metanol. 
Procedimentos gerais de obtenção e identificação dos triterpenos

O fracionamento por cromatografia em coluna (CC) foi conduzido em coluna de vidro, utilizando-se sílica gel 0,063-0,2 mm (Vetec) como fase estacionária e, como sistema eluente, hexano $100 \%$ seguido de misturas de hexano/acetato de etila com aumento da polaridade em escala de 5\%, até chegar ao acetato de etila $100 \%$ e, posteriormente a mistura acetato de etila/metanol, também com aumento de polaridade em escala de $5 \%$ e por último com metanol 100\%. A identificação dos triterpenos puros ou em misturas foram confirmados pelos dados de RMN e, em comparações com os dados da literatura.

$\mathrm{Na}$ cromatografia em camada delgada comparativa (CCD) foram empregadas placas de vidro utilizando sílica gel (60G-MERCK) com indicador de fluorescência $(30 \mathrm{~g})$ com $60 \mathrm{~mL}$ de água destilada como fase estacionária e misturas de hexano, acetato de etila e metanol. As revelações das cromatoplacas foram feitas em câmara de análise de fluorescência por luz ultravioleta, cabine tipo SPECTROLINE (modelo CM-10. Luz tipo SPECTROLINE modelo ENF-260C) pela exposição destas à radiação ultravioleta (UV) nos comprimentos de onda 254 e $366 \mathrm{~nm}$ e por borrifamento com solução ácida de sulfato cérico $\left(\mathrm{CeSO}_{4}\right)$ em ácido sulfúrico $\left(\mathrm{H}_{2} \mathrm{SO}_{4}\right)$ água destilada $1: 1$ ou solução de $\mathrm{H}_{2} \mathrm{SO}_{4}-\mathrm{MeOH}$ 1:1, seguido de aquecimento em estufa até revelação das mesmas; e o critério de pureza adotado para as substâncias isoladas foi a observação de uma única mancha em CCD.

Os espectros de RMN $1 \mathrm{D}\left({ }^{1} \mathrm{H},{ }^{13} \mathrm{C}\right.$ e $\left.1 \mathrm{D}-\mathrm{DEPT}\right)$ foram obtidos em espectrômetro VARIAN modelo MERCURY-300 - 300 MHz) (Laboratório de QuímicaPesquisa, Programa de Pós-Graduação em Química, UFPA). Os deslocamentos químicos de $\mathrm{RMN}{ }^{1} \mathrm{H}$ e ${ }^{13} \mathrm{C}$ foram expressos em ppm $(\delta)$ em relação ao sinal do TMS, como referência interna, e as constantes de acoplamento $(J)$ em Hz.

Bioensaio sensibilidade larvar em papel impregnado
As larvas e fêmeas de $R$. microplus foram cedidas pelo Programa de Pós-Graduação em Ciência Animal, onde também os testes foram realizados. $\mathrm{O}$ procedimento de manutenção das cepas de $R$. microplus em bezerros mestiços está registrado na Comissão de Ética de Uso Animal - CEUA/UFMA, sob protocolo, $\mathrm{n}^{\circ}$ $23115018061 / 2011-01$. O teste de sensibilidade larvar foi realizado de acordo com a técnica desenvolvida por Stone e Haydock ${ }^{35}$ e adaptações da FAO $^{36}$ e Leite $^{37}$. Aproximadamente 100 larvas do carrapato foram colocadas entre dois papéis de filtro medindo 2 × $2 \mathrm{~cm}$ (4 $\mathrm{cm}^{2}$ ), impregnados com $0,3 \mathrm{~mL}$ de cada concentração dos diferentes extratos, fases e compostos puros, os quais formavam um "sanduíche". Esse "sanduíche" foi colocado dentro de um envelope de papel filtro não impregnado de $8,5 \times 8,5 \mathrm{~cm}\left(72,25 \mathrm{~cm}^{2}\right)$, e vedado com pregadores plásticos, segundo FAO Plant Protection Bulletin $^{36}$. O envelope foi acondicionado em estufa a $27^{\circ} \mathrm{C}$ e UR $\geq 80 \%$, durante 24 horas. Após esse período, as larvas vivas e mortas foram contadas com a ajuda de um compressor a vácuo adaptado com uma pipeta, não sendo necessária a contagem quando todas as larvas estavam mortas $(100,0 \%$ de mortalidade) ou vivas $(0,0 \%$ de mortalidade). Foram utilizadas quatro repetições para cada período observado ( 24 horas) para cada tratamento, totalizando 12 pacotes. Os controles foram realizados com água destilada e/ou com as soluções (álcool 70\%; triton 2\%; DMSO 3\%; tween 80 3\%) utilizados na preparação das diferentes soluções de extratos e frações testados (concentrações de 1,$0 ; 5,0 ; 10,0 ; 15,0 ;$ e 20,0\%) e dos compostos puros (concentração $0,5 \%$ ). Os resultados do teste de sensibilidade larvar foram corrigidos de acordo com a fórmula de $\mathrm{Abbott}^{38}$.

\section{Bioensaio sensibilidade das fêmeas ingurgitadas em testes de imersão}

O teste de imersão das fêmeas ingurgitadas de $R$. microplus foi realizado de acordo com a técnica desenvolvida por Drummond e colaboradores ${ }^{39}$. As fêmeas ingurgitadas de $R$. microplus coletadas dos bezerros infestados artificialmente foram lavadas em água 
corrente, secas em papel toalha, e pesadas em grupos de 10 espécimes, com grupos mais homogêneos possíveis. Foram testadas as concentrações de 1,0; 5,0;10,0; 15,0; e $20,0 \%$ de extratos e frações. Cada grupo de carrapato foi submerso durante cinco minutos, nas diferentes concentrações dos extratos, fases e compostos puros das plantas. Após esse período, as fêmeas ingurgitadas foram secas em papel toalha, acondicionadas em placas de Petri e levadas a estufa $\mathrm{BOD}$ a $27^{\circ} \mathrm{C}$ e $\mathrm{UR} \geq 80 \%$, por 15 dias, quando foram coletadas, pesadas as posturas e acondicionadas em seringas descartáveis modificadas para esse uso. As seringas foram acondicionadas novamente para a estufa, $27^{\circ} \mathrm{C}$ e UR $\geq 80 \%$, por mais 15 dias, para a eclosão das larvas, quando foi avaliado visualmente o percentual de eclosão. Os dados como Peso (massa) das Teleóginas (PT), Peso (massa) dos Ovos (PO) e Percentual de Eclosão (\%E), foram avaliados segundo as fórmulas prescritas por Drummond e colaboradores ${ }^{39}$, para o cálculo da Eficiência Reprodutiva (ER) e da Eficiência do Produto (EP) em percentual.

\section{Análise estatística}

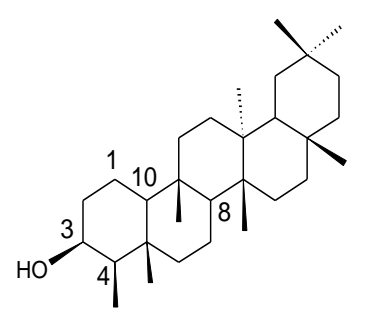

Friedelanol (S1)
O delineamento experimental foi inteiramente casualizado, com quatro repetições, para verificação estatística dos efeitos biológicos, aplicou à técnica estatística Análise de Variância (ANOVA), como os dados satisfizeram as pressuposições, utilizou-se regressão linear simples, utilizando os softwares SPSS 15.0 e BIOESTAT 4.0.

\section{Resultados e discussão}

\section{Estudo químico}

Os triterpenos obtidos e identificados da fração PFQO foram o friedelanol (S1), como composto majoritário, a mistura friedelanol e taraxerol (S2 MISTURA 1), a mistura friedelanol, $\alpha$-amirina (S3) e $\beta$ amirina (S4 - MISTURA 2) e por último a mistura taraxerol, $\alpha$-amirina, $\beta$-amirina e lupeol (S5 - MISTURA 3). Suas fórmulas estruturais de S1-S5 são apresentadas

\section{na FIGURA 2.}

\section{MISTURA 1}

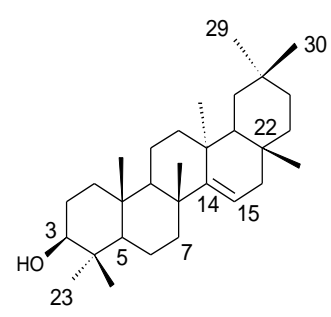

S1 + Taraxerol (S2)

\section{MISTURA 2}

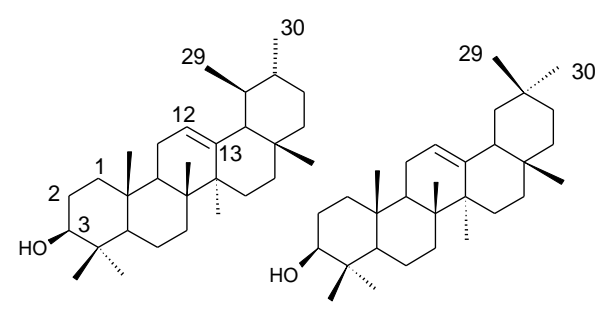

(S1) + alfa-amirina (S3) + beta-amirina (S4)

\section{MISTURA 3}

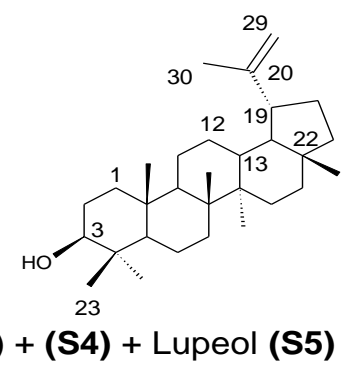

FIGURA 2 - Triterpenos identificados nas folhas espécie Lecythis usitata 
A substância $\mathbf{S 1}$ apresentou-se como cristais e com base nos espectros de $\mathrm{RMN}{ }^{1} \mathrm{H}$ e ${ }^{13} \mathrm{C}$ foi identificada como o friedelanol. O espectro de $\mathrm{RMN}{ }^{1} \mathrm{H}$ da $\mathbf{S 1}$ apresentou vários sinais entre $\delta_{\mathrm{H}} 0,8$ e 1,7 característicos de sinais de hidrogênio metílicos de triterpeno, além de um simpleto largo em $\delta_{\mathrm{H}} 3,74$ de um hidrogênio oximetínico. Esses dados de RMN ${ }^{1} \mathrm{H}$ da $\mathbf{S 1}$ estão de acordo com os encontrados na literatura para o friedelanol ${ }^{40,41}$. Os dados de $\mathrm{RMN}{ }^{13} \mathrm{C}$ da $\mathrm{S} 1$ também estão de acordo com a literatura para friedelanol, com destaque para os valores de $\delta_{\mathrm{C}} 72,73$ atribuídos ao C-3, $\delta_{\mathrm{C}} 61,30$ para o C-10, $\delta_{\mathrm{C}} 53,15$ para o C-8 e $\delta_{\mathrm{C}} 49,13$ para o C-4 (TABELA 1) $^{40,41}$.

A substância $\mathbf{S 2}$ foi identificada como o taraxerol foi obtida em mistura com o friedelanol (MISTURA 1). Destacam-se no espectro de $\mathrm{RMN}{ }^{1} \mathrm{H}$ da mistura $\mathbf{S 1}$ e S2, sinais já mencionados para o friedelanol e os sinais em $\delta_{\mathrm{H}}$ $5,53(d d, J=3,3$ e $8,1 \mathrm{~Hz}, 1 \mathrm{H})$ e em $\delta_{\mathrm{H}} 3,19(d d, J=5,1 \mathrm{e}$ $10,8 \mathrm{~Hz}, 1 \mathrm{H})$, característicos dos hidrogênios H-15 e do H-3, respectivamente, do $\mathbf{S 2}$. Somam-se às informações dos dados dos sinais de $\mathrm{RMN}{ }^{1} \mathrm{H}$ os dados de $\mathrm{RMN}{ }^{13} \mathrm{C}$, além dos sinais já identificados para o friedelanol, os sinais característicos de carbono olefinico $\delta_{\mathrm{C}} 158,02$ e $\delta_{\mathrm{C}}$ 116,83. Todos os sinais do espectro de $\mathrm{RMN}{ }^{13} \mathrm{C}$ da substância obtida (S2) estão de acordo com a literatura ${ }^{42,43}$ para o taraxerol e são apresentados na TABELA 1.

As substâncias $\mathbf{S 3}$ e $\mathbf{S 4}$ foram obtidas em mistura com a substância S1 (MISTURA 2) e identificadas como $\alpha$-amirina e $\beta$-amirina, respectivamente. Os sinais do espectro de $\mathrm{RMN}{ }^{1} \mathrm{H}$ incluem tripletos atribuídos aos hidrogênios H-12 da $\alpha$-amirina (S3) e $\beta$-amirina (S4), em $\delta_{\mathrm{H}} 5,17(J=3,4 \mathrm{~Hz}, 1 \mathrm{H})$ e $\delta_{\mathrm{H}} 5,12(J=3,8 \mathrm{~Hz}, 1 \mathrm{H})$, respectivamente, e em sobreposição $\delta_{\mathrm{H}} 3,31$, atribuído aos hidrogênios oximetínico H-3 e os sinais do friedelanol.

Os sinais no espectro de RMN ${ }^{13} \mathrm{C}$ incluem os sinais do friedelanol e os quatro sinais de carbonos olefínicos $\delta_{\mathrm{C}} 145,10$ e 121,00 e $\delta_{\mathrm{C}} 139,5$ e 124,9 da $\beta$ amirina e $\alpha$-amirina, respectivamente. Todos os sinais atribuídos aos carbonos $\left({ }^{13} \mathrm{C}\right)$ da $\beta$-amirina e $\alpha$-amirina foram comparados com os da literatura ${ }^{12,43}$ e estão na TABELA 1. A substância S5 obtida em mistura com S2, S3 e S4 (MISTURA 3) foi identificada como sendo o triterpeno lupeol. No espectro de $\mathrm{RMN}{ }^{1} \mathrm{H}$ destacam-se os sinais em $\delta_{\mathrm{H}} 4,68(d, J=2,4 \mathrm{~Hz})$ e $\delta_{\mathrm{H}} 4,55(m)$ referentes aos sinais de dois hidrogênios da ligação dupla terminal (H-29) e a sobreposição dos sinais em $\delta_{\mathrm{H}} 3,20$, referente ao sinal do $\mathrm{H}-3$, esses e os demais sinais para o hidrogênio do lupeol estão de acordo com a literatura ${ }^{38,42}$. Segundo Carvalho e colaboradores ${ }^{2}$, os sinais que aparecem como simpleto em aproximadamente $\delta_{\mathrm{H}} 4,50$ e 4,65 , representam os sinais dos dois hidrogênios geminais olefínicos (H-29a e H-29b), esses sinais somados ao deslocamento de hidrogênios de metila em $\delta_{\mathrm{H}} 1,67$, são característicos de sinais de hidrogênio pertencentes a grupamento isopropenil do lupeol. No espectro de RMN ${ }^{13} \mathrm{C}$, os sinais em $\delta_{\mathrm{C}} 150,9$ e 109,3 relativos aos carbonos C-20 e C-29, respectivamente, reforçam as características do grupo olefínico dos triterpenos lupenos. Os dados de ${ }^{13} \mathrm{C}$ obtidos para o lupeol (S5) estão relacionados na TABELA 1 e estão de acordo com a literatura ${ }^{41,44}$. 
TABELA 1- Dados de $\mathrm{RMN}^{13} \mathrm{C}\left(75 \mathrm{MHz}^{13} \mathrm{CDCl}_{3}\right)$ das substâncias obtidas.

\begin{tabular}{|c|c|c|c|c|c|}
\hline C & $\begin{array}{c}\delta^{13} \mathrm{C}-\text { Friedelanol } \\
\text { (S1) }\end{array}$ & $\begin{array}{c}\delta^{13} \mathrm{C} \text { Taraxerol } \\
(\mathrm{S} 2)\end{array}$ & $\begin{array}{c}\delta^{13} \mathrm{C}-\alpha \text {-amirina } \\
(\mathrm{S3})\end{array}$ & $\begin{array}{c}\delta^{13} \mathrm{C} \text { - } \beta \text {-amirina } \\
\text { (S4) }\end{array}$ & $\begin{array}{c}\delta^{13} \mathrm{C} \text { Lupeol } \\
(5)\end{array}$ \\
\hline 1 & 15,7 & 37,9 & 38,7 & 38,5 & 38,7 \\
\hline 2 & 36,0 & 27,0 & 26,8 & 27,2 & 27,4 \\
\hline 3 & 72,7 & 79,0 & 79,0 & 79,0 & 79,0 \\
\hline 4 & 49,1 & 39,2 & 38,7 & 38,7 & 38,7 \\
\hline 5 & 37,7 & 55,5 & 55,1 & 55,1 & 55,1 \\
\hline 6 & 41,6 & 18,7 & 18,3 & 18,3 & 18,3 \\
\hline 7 & 17,5 & 41,2 & 32,6 & 32,8 & 34,2 \\
\hline 8 & 53,1 & 38,7 & 39,7 & 39,9 & 40,8 \\
\hline 9 & 37,0 & 48,6 & 47,5 & 47,6 & 50,4 \\
\hline 10 & 61,3 & 37,6 & 36,9 & 36,8 & 37,1 \\
\hline 11 & 35,3 & 17,4 & 23,5 & 23,2 & 20,9 \\
\hline 12 & 30,6 & 35,4 & 121,7 & 124,3 & 25,1 \\
\hline 13 & 38,3 & 38,1 & 145,1 & 139,5 & 38,0 \\
\hline 14 & 39,6 & 158,0 & 41,6 & 41,5 & 42,8 \\
\hline 15 & 32,2 & 116,8 & 26,1 & 28,7 & 27,4 \\
\hline 16 & 35,5 & 36,6 & 27,1 & 26,5 & 35,5 \\
\hline 17 & 29,9 & 37,7 & 32,4 & 33,7 & 42,9 \\
\hline 18 & 42,7 & 49,2 & 47,1 & 59,0 & 48,2 \\
\hline 19 & 53,1 & 35,1 & 46,7 & 39,5 & 47,9 \\
\hline 20 & 28,1 & 28,7 & 31,0 & 39,6 & 150,9 \\
\hline 21 & 32,7 & 33,6 & 34,6 & 31,2 & 29,8 \\
\hline 22 & 39,2 & 33,0 & 37,1 & 41,5 & 39,9 \\
\hline 23 & 11,6 & 27,9 & 28,9 & 28,1 & 28,0 \\
\hline 24 & 16,3 & 15,7 & 15,5 & 15,6 & 15,4 \\
\hline 25 & 18,2 & 15,3 & 15,4 & 15,6 & 16,1 \\
\hline 26 & 20,1 & 29,8 & 16,7 & 16,8 & 15,9 \\
\hline 27 & 18,6 & 25,8 & 25,9 & 23,3 & 14,5 \\
\hline 28 & 32,0 & 29,7 & 28,3 & 28,7 & 18,0 \\
\hline 29 & 35,0 & 33,3 & 33,3 & 17,4 & 109,2 \\
\hline 30 & 31,7 & 21,2 & 23,6 & 21,3 & 19,3 \\
\hline
\end{tabular}

$\mathrm{C}=$ carbono; $\delta^{13} \mathrm{C}=$ Deslocamento químico do carbono treze; $(\mathrm{S} 1)=$ friedelanol; $(\mathrm{S} 2)=$ taraxerol; $(\mathrm{S} 3)=\alpha$-amirina $;(\mathrm{S} 4)$ $=\beta$-amirina; (S5) = lupeol. 
Os esqueletos triterpênicos têm 30 átomos carbonos e são estruturalmente muito diversificados e são de grande interesse devido às diversas atividades biológicas apresentadas, servindo como candidatos ou protótipos de novos fármacos, no entanto, o papel biológico de diversos terpenoides ainda não é totalmente conhecido $^{46}$

No mundo ocidental, uma média de $250 \mathrm{mg}$ de triterpenos são ingeridos por dia, em grande parte presentes em óleos vegetais, cereais, frutas e vegetais presentes na alimentação humana. Durante a última década pelo menos 25 estudos clínicos, 20 patentes foram depositados e, pelo menos, dez princípios ativos à base de triterpenos são comercializados em todo o mundo ${ }^{47}$.

De acordo com trabalhos realizados por Silva; Duarte; Vieira Filho ${ }^{46}$, os triterpenos pentacíclicos são responsáveis pela atividade no tratamento de queratose actínica. As pomadas são constituídas de, aproximadamente, $87 \%$ de triterpenos, tais como, a betulina (80\%), ácido betulínico (3\%) e lupeol (2\%).

Ainda, sobre o lupeol que pode ser encontrado no repolho branco, pimenta verde, morango, azeite de oliva verde, mangas e uvas foram relatados possuir efeitos benéficos como um agente terapêutico e preventivo para uma variedade de desordens, entre elas, potencial antiinflamatório como quimiopreventivo e agente quimioterapêutico e que em sua dose terapêutica eficaz não apresenta toxicidade para as células e tecidos normais ${ }^{47}$.

Em outra pesquisa ficou demonstrado à atividade captadora de radicais livres dos extratos de folhas de Ficus pseudopalma usando testes bioquímicos e o triterpeno lupeol foi identificado como um dos os possíveis componentes ativos com base em cromatográfica e análise de espectroscopia de infravermelho. Esses resultados significativos têm demonstrado a importância da triagem fitoquímica de flora endêmica que, potencialmente, abriga farmacologicamente candidatos promissores de novas drogas ${ }^{48}$ bem como em efeitos sinérgicos associados a antibióticos no combate a listeriose ${ }^{49}$.

\section{Bioensaios carrapaticidas}

Neste trabalho, é apresentado o primeiro relato de estudo de atividade biológica da espécie L. usitata. Os gráficos de regressão linear da atividade carrapaticida dos extratos e fração das folhas de L. usitata ativos sobre larvas e fêmeas ingurgitadas de $R$. microplus estão na FIGURA 3. O extrato hexânico PFQA não apresentou eficiência carrapaticida nas concentrações testadas. O extrato metanólico, PFQP apresentou $91 \%$ de mortalidade, na concentração de $20 \%$. A fração PFQO obtida da partição do extrato polar apresentou mortalidade de $94 \%$ e $97 \%$, nas concentrações de $15 \%$ e 20\%, respectivamente. O extrato metanólico PFQP e a fração PFQO também foram testados sobre fêmeas ingurgitadas da mesma espécie de carrapato, e os resultados dos dois bioensaios com larvas e fêmeas do PFQP apresentaram resultados próximos, o ajustamento dos dados a um modelo de regressão linear resultou nas equações $\mathrm{Y}=$ $3,9024 \mathrm{x}-7,8367\left(\mathrm{R}^{2}=76,96\right)$ e $\mathrm{Y}=4,1654 \mathrm{x}-9,5722\left(\mathrm{R}^{2}\right.$ $=$ 77,58), respectivamente, para larvas e fêmeas (FIGURA 3).

\section{A fração PFQO apresentou atividade}

carrapaticida, contudo, os dados verificados para as fêmeas foram inferiores ao apresentado para as larvas, o ajustamento dos dados a um modelo de regressão linear resultou nas equações $\mathrm{Y}=5,4378 \mathrm{x}-11,7213\left(\mathrm{R}^{2}=\right.$ $79,25)$ e $Y=1,6472 x-5,3349\left(R^{2}=67,57\right)$, respectivamente, para larvas e fêmeas (FIGURA 3). 

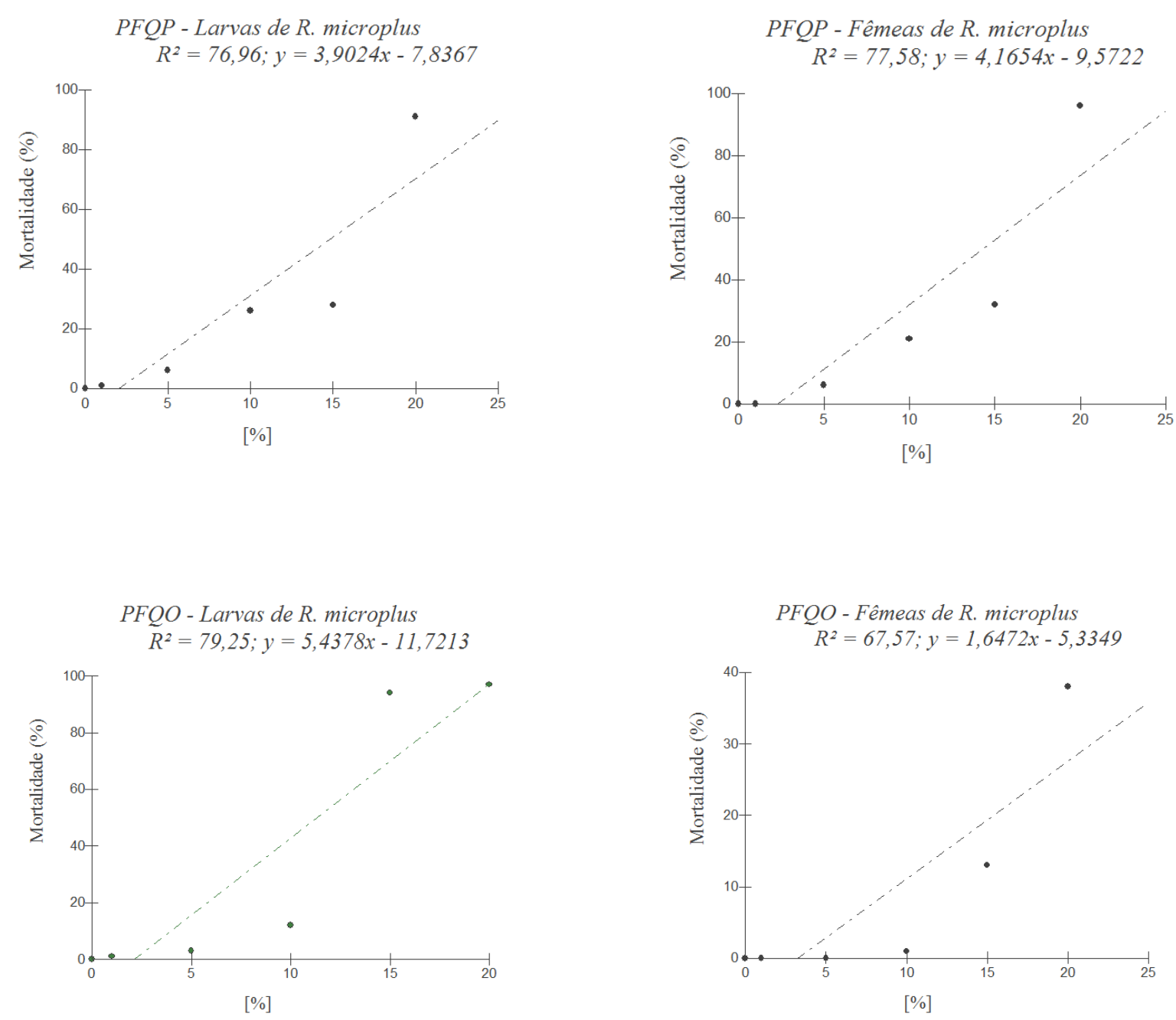

FIGURA 3 - Gráficos de regressão linear da atividade carrapaticida dos extratos e frações de Lecythis usitata (PFQP = Extrato polar metanolico; PFQO = fração orgânica acetato de etila ; $\mathrm{R}^{2}=$ coeficiente de determinação).

Os estudos de atividade biológica com plantas seguem em linhas gerais os testes com extratos brutos e frações de acordo com metodologias variadas, contudo, a etapa seguinte é testar os metabólitos obtidos, isoladamente ou em técnicas que verifiquem um possível o efeito sinérgico ${ }^{50}$.

Os triterpenos pentacíclicos obtidos nessa investigação e em mistura foram analisados em bioensaios com larvas e fêmeas de $R$. microplus e nas concentrações avaliadas não apresentaram atividade larvicida.

Contudo, em estudo, o triterpeno pentacíclico taraxerol obtido da espécie Excoecaria agallocha, na concentração a $2,0 \%$ apresentou $80 \%$ de mortalidade das larvas da lagarta Helicoverpa armigera com $\mathrm{LC}_{50}$ de
0,0001 $\mathrm{g} \mathrm{kg}^{-1}$ de peso corporal. Estudos da espécie citada sugere a possibilidade de formular um potencial pesticida natural de E. agallocha contra pragas lepidópteras ${ }^{51}$.

O uso de biopesticidas e produtos de gestão alternativa vêm aumentando e esse progresso é ilustrado pelo número de registros de biopesticidas e pesquisas relacionadas ao tema e podem apresenar grandes oportunidades comerciais $^{52}$. Como exemplo de substâncias bioativas, tem-se a rotenona, substância que ocorre naturalmente em certas plantas do gênero Derris, Lonchocarpus e Tephrosia. É usada em todo o mundo como um pesticida de amplo espectro (acaricida, inseticida e piscicida) ${ }^{53}$. 
Importante também são as lactonas macrocíclicas; Wang e colaboradores ${ }^{54}$ isolaram uma lactona macrocíclica a partir do caldo de fermentação de Streptomyces microflavus que apresentou atividade nematicida contra Caenorhabditis elegans $\left(\mathrm{LC}_{50}=17,4\right.$ $\left.\mu \mathrm{g} \mathrm{mL} L^{-1}\right)$.

Em outra pesquisa os resultados indicam que as formulações hidroetanólicos de timol têm atividade acaricida sobre larvas de $R$. sanguineus e larvas de Dermacentor nitens, provocando mortalidade superior a $90 \%$, após $24 \mathrm{~h}$ na concentração de $10 \mathrm{mg} \mathrm{mL}^{-1}$ para as duas espécies de carrapato estudadas ${ }^{55}$.

Estudo sobre as folhas de Protium spruceanum apresentado por Figueiredo e colaboradores ${ }^{56}$ identificou catequinas, flavonoides e $\beta$-amirina, os quais não foram eficazes contra fêmeas ingurgitadas de $R$. microplus, no entanto os extratos reduziram em $90 \%$ a eclosão das larvas na concentração de $100 \mathrm{mg} \mathrm{mL}^{-1}$ e mortalidade larval acima de $88 \%$ a $100 \mathrm{mg} \mathrm{mL}^{-1}$, representando um agente alternativo para controle integrado de carrapato.

Já a atividade carrapaticida da espécie Uraria picta, foi atribuída a diferentes classes de compostos químicos encontrados, como os fenólicos, flavonoide, esterol, e derivados de terpeno ${ }^{57}$. Análise em CG-EM dos extratos metanólicos de caules e de folhas de Petiveria alliacea demonstrou que as amostras ativas continham principalmente o dissulfeto de dibenzila e o trissulfeto de dibenzila, a mortalidade de $100 \%$ no bioensaio de teste de imersão de larvas de $R$. microplus pode ser atribuída aos dois compostos $\operatorname{citados}^{58}$ de acordo com os autores.

O fracionamento do extrato bioativo de Piper guanacastensis sobre Aedes atropalpus proporcionou a obtenção do 4-hidróxi-3-(3'-metil-2'-butenil)benzoato de metila como o principal constituinte $\left(\mathrm{LC}_{50} 20,5 \mu \mathrm{g} \mathrm{mL}^{-1}\right)^{59}$.

Segundo Viegas $\mathrm{Jr}^{60}{ }^{60}$, estudos relativos à ação e ao impacto ambiental dessas substâncias que podem ser biodegradáveis e seletivas para parasitos indesejados têm conduzido à avaliação e descoberta de uma série de moléculas, principalmente terpenos e alcaloides, candidatos a biopraguicidas e são uma alternativa eficaz com menor impacto sobre a saúde humana, dos animais domésticos e para o ambiente.
Outro aspecto importante é a divulgação de informações sobre a espécie estudada, possibilitando o conhecimento dessa importante planta do cerrado maranhense, auxiliando a sua conservação, pois, ainda é pouco estudada e a divulgação e busca de extratos e metabólitos secundários de plantas a fim de encontrar potenciais carrapaticidas, fitoterápicos e fármacos de aplicação futura.

\section{Conclusões}

O primeiro relato de estudo de atividade biológica das folhas da espécie Lecythis usitata sobre larvas e fêmeas ingurgitadas de Rhipicephalus microplus, mostrou que extrato metanólico, PFQP e a fração PFQO apresentaram ação carrapaticida com mortalidade das larvas superior a 90\%, na concentração de $20 \%$. Dados semelhantes foram observados para os bioensaios com fêmeas ingurgitadas, com exceção da fração PFQO, que apresentou atividade carrapaticida inferior ao verificado para as larvas. O extrato hexânico não apresentou atividade carrapaticida.

No estudo químico da fração PFQO obtida das folhas da espécie Lecythis usitata, foram identificados triterpenos pentacíclicos, friedelanol, como composto majoritário, a mistura friedelanol e taraxerol, a mistura friedelanol, $\alpha$-amirina e $\beta$-amirina e por último a mistura taraxerol, $\alpha$-amirina, $\beta$-amirina e lupeol. $\mathrm{O}$ friedelanol e as misturas de triterpenos também não apresentaram atividade carrapaticida.

\section{Declaração de conflitos de interesses}

Os autores do artigo afirmam que não houve nenhuma situação de conflito de interesse, tais como propostas de financiamento, emissão de pareceres, promoções ou participação em comitês consultivos ou diretivos, entre outras, que pudessem influenciar no desenvolvimento do trabalho.

\section{Agradecimentos}

Os autores agradecem a Universidade federal do Pará, ao Centro de Ciências Agrárias e Ambientais da 
Universidade Federal do Maranhão pelo suporte de infraestrutura e a FAPESPA e FAPEMA pelo suporte financeiro.

\section{Referências}

1. THIMMAPPA, R.; GEISLER, K.; LOUVEAU, T.; O'MAILlE, P.; OSBOURN, A. Triterpene biosynthesis in plants. Annual Review of Plant Biology, v. 65, p. 225-257, 2014.

2. CARVALHO, M. G. de; VELANDIA, J. R.; OLIVEIRA, L. F. de; BEZERRA, F. B. Triterpenos isolados de Eschweilera longipes Miers (Lecythidaceae). Química Nova, v. 21, n. 6, p. 740743, 1998.

3. CRUBLET, M.-L; POUNY, I; DELAUDE, C; LAVAUD, C. Acylated triterpenoid sapanins from the Stem Bark of Foetidia Africana. Journal of Natural Products, n. 65, n. 11, p. 1500-1567, 2002.

4. ALMEIDA, M. de F. O; MELO, A. C. R. de; PINHEIRO, M. L. B.; SILVA, J. R. de A.; SOUZA, A. D. L. de S. Constituintes químicos e atividade leishmanicida de Gustavia elliptica (Lecythidaceae). Química Nova, v. 34, n. 7, p. 1182-1187, 2011.

5. FRANCO, E. A. P.; BARROS, R. F. M. Uso e diversidade de plantas medicinais no Quilombo Olho d'água dos Pires, Esperantina, Piauí. Revista Brasileira de Plantas Medicinais, v. 8, n. 3, p. 7888, 2006.

6. HUSSIN, N. M.; MUSE, R.; AHMAD, S.; RAMLI, J.; MAHMOOD, M.; SULAIMAN, M. R.; SHUKOR, M.Y.A.; RAHMAN, M. F. A.; AZIZ, K. N. K. Antifungal activity of extracts and phenolic compounds from Barringtonia racemosa $\mathrm{L}$. (Lecythidaceae). African Journal of Biotechnology, v. 8, p. 2835-2842, 2009.

7. PATIL, K. R.; PATIL, C. R.; JADHAV, R. B.; MAHAJAN, V. K.; PATIL, P. R.; GAIKWAD, P. S. Anti-Arthritic activity of bartogenic acid Isolated from fruits of Barringtonia racemosa Roxb. (Lecythidaceae). Evidence-Based Complementary and Alternative Medicine, v. 5, n. 1, p. 112-117, 2011.

8. McRAE, J. M.; YANG, Q.; CRAWFORD, R. J.; PALOMBO, E. A. Antibacterial compounds from Planchonia careya leaf extracts. Journal of Ethnopharmacology, v. 116, n. 3, p. 554-560, 2008.

9. PINHEIRO, M. M. G.; BESSA, S. O.; FINGOLO, C. E.; KUSTER, R. M.; MATHEUS, M. E.; MENEZES, F. S.; FERNANDES, P. D. Antinociceptive activity of fractions from Couroupita guianensis Aubl. leaves. Journal of Ethnopharmacology, v. 127, n. 2, p. 407-413, 2010.

10. DUARTE, E. F.; SANTOS, J. A. dos; ALMEIDA, D. S.; SANTOS, C. H. B.; AZEVEDO NETO, A. D. de; CRUZ, C. R. P.; PEIXOTO, C. P. Maturação de frutos e sementes de inhaíba (Lecythis lurida [Miers]). Revista de Biologia Neotropical, v. 17, n. 1, p. 15-34, 2020.

11. RISSINI, C. T. Árvores e madeiras úteis do Brasil: manual de dendrologia brasileira/Carlos Toledo Rizzini - 2 ${ }^{\text {a }}$ Ed. - São Paulo: Edgard Blucher, 1978.

12. SOUZA, A. D. L de; ROCHA, A. F. I. da, PINHEIRO, M. L. B., ANDRADE, C. H. de S., GAlOTTA, A. L. de A. Q., SANTOS, M. do P. S. S. Constituintes químicos de Gustavia augusta $\mathrm{L}$. (lecythidaceae). Química Nova, v. 24, n. 4, p. 439442, 2001.

13. HUANG, Y.-Y.; MORI, S. A.; KELLY, L. M. Toward a phylogenetic-based generic classification of neotropical Lecythidaceae-I. Status of Bertholletia, Corythophora, Eschweilera and Lecythis. Bertholletia. Phytotaxa, v. 203, n. 2, p. 085-121, 2015.

14. MORI, S. A.; TSOU, C. H.; WU, C. C.; CRONHOLM, B.; ANDERBERG, A. A. Evolution of Lecythidaceae with an emphasis on the circumscription of neotropical genera: Information from combined NDHF and TRNL-F sequence data. 
American Journal of Botany, v. 94, n. 3, p. 289301, 2007.

15. SOUZA, V. C.; LORENZI, H. Botânica

sistemática: Guia ilustrativo para identificação das famílias de angiospermas da flora brasileira, baseado em APG II, São Paulo, Nova Odessa, SP, 2005.

16. PEREIRA, J. R.; FAMADAS, K. M. Avaliação "in vitro" da eficiência do extrato da raiz do Timbó (Dahlstedtia pentaphylla) (Leguminosae, Papilionoidae, Millettiedae) sobre Boophilus microplus (CANESTRINI, 1887) na Região do Vale do Paraíba, São Paulo, Brasil. Arquivo Instituto Biológico, v.71, n. 4, p.443-450, 2004.

17. PEREIRA, J. R.; FAMADAS, K. M. The efficiency of extracts of Dahlstedtia pentaphylla (Leguminosae, Papilionoidae, and Millettiedae) on Boophilus microplus (Canestrini, 1887) in artificially infested bovines. Veterinary Parasitology, v. 142, n. 1-2, p. 192-195, 2006.

18. BROGLIO-MICHELETTI, S. M. F.; DIAS, N. da S.; VALENTE, E. C. N.; SOUZA, L. A. de; LOPES, D. O. P.; SANTOS, J. M. dos. Ação de extrato e óleo de nim no controle de Rhipicephalus (Boophilus) microplus (Canestrini, 1887) (Acari: Ixodidae) em laboratório. Revista Brasileira de Parasitologia Veterinária, v. 19, n. 1, p. 44-48, 2010.

19. NAWAZ, M.; SAJID, S. M.; AHMED, Z.; WAQAS, M.; AHMED, T.; HUSSAIN, A.; MOHIUD-DIN, A.; SHAMIM, A.; ZUBAIR, M.; KHAL, I. Anti-Tick Activity of leaves of Azadirachta indica, Dalbergia sisso and Morus alba against Rhipicephalus microplus (Acari: Ixodidae). Acta Parasitologica Globalis, v. 6, n. 1, p. 60-64, 2015.

20. CHAGAS, A. C. de S.; PASSOS, W. M.; PRATES, H. T.; LEITE, R. C.; FURLONG, J.; FORTES, I. C. P. Efeito acaricida de eucalyptus em Boophilus microplus: óleos essenciais e concentrados emulsionáveis. Brazilian Journal of Veterinary Research and Animal Science, v. 39, n. 5, p. 247 253, 2002.
21. CHAGAS, A. C. de S.; BARROS, L. D. de; COTINGUIBA, F.; FURLAN, M.; GIGLIOTI, R.; OLIVEIRA, M. C. de S.; BIZZO, H. R. In vitro efficacy of plant extracts and synthesized substances on Rhipicephalus (Boophilus) microplus (Acari: Ixodidae). Parasitology Research, v. 110, n. 1, p. 295-303, 2012.

22. FERNANDES, F. de F.; FREITAS, E. de P. e S.; COSTA, A. C. Da; SILVA, I. G. Da. Larvicidal potential of Sapindus saponaria to control of the cattle tick, Boophilus microplus. Pesquisa Agropecuária Brasileira, v. 40, n. 1, p. 12431245, 2005.

23. MARTINS, R. M. Estudio in vitro de la acción acaricida del aceite esencial de la gramínea Citronela de Java (Cymbopogon winterianus) en la garrapata Boophilus microplus. Revista Brasileira de Plantas Medicinais, v. 8, p. 71-78, 2006.

24. HEIMERDINGER, A.; OLIVO, C. J.; MOLENTO, M. B.; AGNOLIM, C. A.; ZIECH, M. F.; SCARAVELLI, L. F. B.; SKONISKI, F. R.; BOTH, J. F.; CHARÃO, P. S. Extrato alcoólico de Capim-cidreira (Cymbopogon citratus) no controle do Boophilus microplus em bovinos. Revista Brasileira Parasitologia. Veterinária, v. 15, n. 1, p. 37-39, 2006.

25. LIMA, A. da SILVA; SOUSA FILHO, J. G. do N.; PEREIRA, S. G.; GUILHON, G. M. S. P.;

SANTOS, L. S.; COSTA JUNIOR, L. M. Acaricide activity of different extracts from Piper tuberculatum fruits against Rhipicephalus microplus. Parasitol Research, v. 113, p. 107-113, 2014.

26. AMORIM, A. R. de; BUCHINI, J. L. C.; MARZOLLA, I. P. MARTINS, G. C. G.; GOBETTI, S. T. C.; MARÇAL, W. S. O uso irracional de medicamentos veterinários: uma análise prospectiva. Revista Brasileira de Higiene e Sanidade Animal, v. 14, n. 2, p. 196-205, 2020.

27. GRISOLIA, C. K. Agrotóxicos-mutações, reprodução e câncer. Brasília- DF: Editora Universidade de Brasília, 2005. 
28. GRISI, L.; MASSARD, C. L.; MOYA B. G. E.;

PEREIRA, J. B. Impacto econômico das principais ectoparasitoses em bovinos no Brasil. A Hora

Veterinária, v. 125, n. 21, p. 8-10, 2002.

29. SILVA, T. P. P. da; MOREIRA, J. C.; PERES, F. Serão os carrapaticidas agrotóxicos? Implicações na saúde e na percepção de riscos de trabalhadores da pecuária leiteira. Ciência \& Saúde Coletiva, v. 17, n. 2, p. 311-325, 2012.

30. ESTRADA-PEÑA, A.; SZABÓ, M.; LABRUNA, M; MOSQUEDA, J.; MERINO, O.; TARRAGONO, E.; VENZAL, J. M.; LA FUENTE, J. de. Towards an effective, rational and sustainable approach for the control of cattle ticks in the Neotropics. Vaccines, v. 8, n. 9, p. 2-9, 2020.

31. DOMÍNGUEZ-GARCÍA, D. I.; ROSARIO-CRUZ, R.; ALMAZÁN-GARCÍA, C.; OAXACA, J. A. S; DE LA FUENTE, J. Boophilus microplus: Aspectos Biológicos y moleculares de la resistencia a los acaricidas y su Impacto en la salud animais.

Tropical and Subtropical Agroecosystems, v. 12, 181-192, 2010.

32. FAZA, A. P.; PINTO, I. S.; FONSECA, I.; ANTUNES, G. R.; MONTEIRO, C. M.; DAEMON, E.; MUNIZ, M. de S.; MARTINS, M. F.; FURLONG, J.; PRATA, M. C. A new approach to characterization of the resistance of populations of Rhipicephalus microplus (Acari: Ixodidae) to organophosphate and pyrethroid in the state of Minas Gerais, Brazil. Experimental Parasitology, v. 134 , n. 4, p. 519-23, 2013.

33. LARA, T. I. da C. de; GARCIA, S. D. O impacto do uso dos agrotóxicos na saúde pública: revisão de literatura. Revista Saúde e Desenvolvimento

Humano, v. 8, n. 1, p. 85-96, 2020.

34. GRISI, L.; LEITE, R. C.; MARTINS, J. R. de S.; BARROS, A. T. M. de; ANDREOTTI, R.; CANÇADO, P. H. D.; LEÓN, A. A. P. de; PEREIRA, J. B.; VILLELA, H. S. Reassessment of the potential economic impact of cattle parasites in Brazil. Revista Brasileira Parasitologia.

Veterinária, v. 23, n. 2, p. 150-156, 2014.
35. STONE, B. F.; HAYDOCK, K. P. A method for measuring the acaricide susceptibility of the cattle Boophilus microplus (Can.). Bulletin of Entomological Research, v. 53, p. 563-578, 1962.

36. FAO. Plant Protection Bulletin. Recommended methods for the detection and measurement of resistance of agricultural pests to pesticides. Tentative methods for larvae of cattle tick Boophilus spp. FAO method, v.19, n. 7, p.15-18, 1971.

37. LEITE, R. C. Boophilus microplus (Canestrini, 1887): susceptibilidade, uso atual e retrospectivo de carrapaticidas em propriedades das regiões fisiogeográficas da baixada do Grande-Rio e Rio de Janeiro. Uma abordagem epidemiológica. Belo Horizonte - Minas Gerais - Brasil. 1988. $151 \mathrm{f}$. Tese (Doutorado em Medicina Veterinária Preventiva), Curso de Pós-graduaçãoem Medicina Veterinária, Universidade Federal de Minas Gerais. 1987.

38. ABBOTT, W. S. A method of computing the effectiveness of an insecticide. Journal of Economic Entomology, v. 18, n. 1, p. 265-267, 1925.

39. DRUMMOND, R. O.; ERNEST, S. E.; TREVINO, J. L.; GLADNEY, W. J.; GRAHAM, O. H. Boophilus annulatus and Boophilus microplus: laboratory tests for insecticides, Journal of Economy Entomolology, v. 66, n. 1, p. 130-133, 1973.

40. BEGUM, F.; HAQUE, M. R.; NAHAR, K. S.; RASHID, M. A. Secondary metabolites from different extractives of Stereospermum suaveolens.

\section{Dhaka University Journal of Pharmaceutical}

Sciences, v. 13, n. 1, p. 31-36, 2014.

41. ISLAM, R.; AHMED, I.; SIKDER, A. A.; HAQUE, M. R.; MANSUR, A. A.; AHMED, M.; RASHEED, M.; RASHID, M. A. Chemical investigation of Mesua nagassarium (Burm. f.) Kosterm. Journal of Basic \& Applied Sciences, v. 10, p. 124-128, 2014. 
42. MUH, L.; AL MUGARRABUN, R.; AHMAT, N.; ARIS, S. R. S.; NORIZAN, N. Phytochemical investigation of the stem bark of Sapium baccatum (Roxb.). Australian Journal of Basic and Applied Sciences, n. 8, v. 3, p. 432-438, 2014.

43. CASTORENA, A.-L. P. Triterpenes and other metabolites from Tibouchina urvilleana. Journal of Mexican Chemical Society, v. 58, n. 2, p. 218$222,2014$.

44. CURSINO, L. M. de C.; MESQUITA, A. S. S.; MESQUITA, D. W. de O.; FERNANDES, C. C.; PEREIRA JUNIOR, O. L.; AMARAL, I. L. do; NUNEZ, C. V. Triterpenos das folhas de Minquartia guianensis Aubl. (Olacaceae). Acta Amazônica, v. 39, n. 1, p. 181-186, 2009.

45. ISLAM, R.; AHMED, I.; SIKDER, A. A.; HAQUE, M. R.; MANSUR, A. A.; AHMED, M.; RASHEED, M.; RASHID, M. A. Chemical investigation of Mesua nagassarium (Burm. f.) Kosterm. Journal of Basic \& Applied Sciences, v. 10, p. 124-128, 2014.

46. SALEEM, M. Lupeol, a novel anti-inflammatory and anti-cancer dietary triterpene. Cancer Letters, v. 285, n. 2, p. 109-115, 2009.

47. SILVA, F. C.; DUARTE, L. P.; VIEIRA FILHO, S. A. Celastráceas: fontes de triterpenos pentacíclicos com potencial atividade biológica. Revista Virtual de Química, v. 6, n. 5, p. 1205-1220, 2014.

48. SANTIAGO, L.; A.; MAYOR, A. B. R. Lupeol: An antioxidant triterpene in Ficus pseudopalma Blanco (Moraceae). Asian Pacific Journal of Tropical Biomedicine, v. 4, n. 2, p. 109-118, 2014. 49. PENDUKA, D.; MOSA, R.; SIMELANE, M.; BASSON, A.; OKOH, A.; OPOKU, A. Evaluation of the anti-Listeria potentials of some plant-derived triterpenes. Annals of Clinical Microbiology and Antimicrobials, v. 13, n. 37, p. 2-7, 2014.

50. SANTHANAM, S. R.; SUBRAMANIAN, M.; EGIGU, M. C.; PARIDA, A. Pentacyclic triterpenoids and a linear alkane from the Milky mangrove tree (Excoecaria agallocha L.) are toxic to the larva of Helicoverpa armigera Hubner.
(Lepidoptera: Noctuidae). International Journal of Advanced Research, v. 2, n. 6, p. 1-12, 2014.

51. SCHENKEL, E. P.; GOSMANN, G; PETROVIC, P. R. Produtos de origem vegetal e o desenvolvimento de medicamentos. In: SIMÕES, C. M. O.; SCHENKEL, E. P.; GOSMANN, G.; MELLO, J. C. P.; MENTZ, L. A.; PETROVICK, P. R. (org.) Farmacognosia: da planta ao medicamento. 5 ed. ver. amp., Porto Alegre: UFRGS, Florianópolis: UFSC, 2003.

52. SEIBER, J. N.; COATS, J.; DUKE, S. O.; GROSS, A. D. Biopesticides: state of the art and future opportunities. Journal of Agricultural and Food Chemistry, v. 62, p. 11613-11619, 2014.

53. GUPTA, R. C. Capítulo 52 - Rotenone. Veterinary Toxicology, $2^{\mathrm{a}}$ ed., 2012, p. 620-623.

54. WANG, X. J.; ZHANG, J.;LIU, C. X.; GONG, D. L.; ZHANG, H.; WANG, J. D.; YAN, Y. J.; XIANG, W. S. A novel macrocyclic lactone with insecticidal bioactivity from Streptomyces microflavus neau3. Bioorganic \& Medicinal Chemistry Letters, v. 21, n. 18, p. 5145-5148, 2011.

55. DAEMON, E.; MATURANO, R.; MONTEIRO, C. M.; GOLDNER, M. S.; MASSONI, T. Acaricidal activity of hydroethanolic formulations of thymol against Rhipicephalus sanguineus (Acari: Ixodidae) and Dermacentor nitens (Acari: Ixodidae) larvae. Veterinary Parasitology, v. 186, n. 3-4, p. 542$545,2012$.

56. FIGUEIREDO, J. C. G.; NUNES, Y. R. F.; VASCONCELOS, V. de O.; ARRUDA, S. R.; MORAIS-COSTA, F.; SANTOS, G. S. C.; ALVEZ, F. S.; DUARTE, E. R. Effects of leaf extracts of Protium spruceanum against adult and larval Rhipicephalus microplus. Experimental and applied acarology, v. 79, p. 447-458, 2019.

57. IGBOECHI, A. C.; OSAZUWA, E.O.; IGWE, U.E. Laboratory evaluation of the acaricidal properties of extracts from Uraria picta (Leguminosae). Journal of Ethnopharmacology, v. 26, n. 3, p. 293-298, 1989. 
58. ROSADO-AGUILAR, J. A.; AGUILAR-

CABALLERO, A.; RODRIGUEZ-VIVAS, R. I.;

BORGES-ARGAEZ, R.; GARCIA-VASQUEZ, Z.;

MENDEZ-GONZALEZ, M. Acaricidal activity of

extracts from Petiveria alliacea (Phytolaccaceae)

against the cattle tick, Rhipicephalus (Boophilus)

microplus (Acari: ixodidae). Veterinary

Parasitology, v. 168, n 3-4, p. 299-303, 2010.

59. PEREDA-MIRANDA, R.; BERNARD, C. B.;

DURST, T.; ARNASON, J. T.; SÁNCHEZ-

VINDAS, P.; POVEDA, L.; SAN ROMÁN, L.

Methyl 4-Hydroxy-3-(3'-methyl-2'-

butenyl)benzoate, major insecticidal principle from

Piper guanacastensis. Journal of Natural

Products, v. 60, n. 3, p. 282-284, 1997.

60. VIEGAS JR., C. Terpenos com atividade inseticida: uma alternativa para o controle químico de insetos.

Quimica Nova, v. 26, n. 3, p. 390-400, 2003. 